\title{
Formation and Characterisation of Mn-MIL-100
}

\author{
Helge Reinsch $^{a}$ and Norbert Stock ${ }^{a, *}$ \\ Received (in $X X X, X X X)$ Xth $X X X X X X X X X 20 X X$, Accepted Xth $X X X X X X X X X 20 X X$ \\ DOI: 10.1039/b000000x
}

\begin{abstract}
5 The synthesis and characterisation of the Mn-based analogue of the MIL-100-framework is reported. The title compound is synthesised from a methanolic solution of trimesic acid and manganese(II) nitrate in very short reaction times. During the reaction, the $\mathrm{Mn}^{2+}$-ions are oxidized in-situ to $\mathrm{Mn}^{3+}$-ions. The obtained MOF was characterised by XRPD-measurements, IR-spectroscopy, thermogravimetric measurements and sorption experiments. To have a closer look at the crystallisation occurring after the in 10 situ metal oxidation-reaction that results in the formation of the title compound, in-situ EDXRPDexperiments under solvothermal conditions were carried out. These time-resolved measurements could be evaluated by two different kinetic models (by Avrami and Gualtieri) for crystallisation. The results indicate a two stage reaction process, which are dominated by different reaction mechanisms.
\end{abstract}

\section{Introduction}

15 In the field of porous materials, metal-organic frameworks (MOFs) represent a rather young but still rapidly growing class of compounds. ${ }^{1}$ These materials are in general based on metal ions or cationic metal-oxo-clusters which are connected via anionic organic moieties, mostly carboxylate or phosphonate but also 20 sulfonate or imidazolate ions. The metal-organic materials often exhibit highly desirable properties. Thus, large specific surface areas as well as unique sorption properties were reported. ${ }^{2}$ In some cases flexible framework structures or chemically specific sorption behaviour can be observed. ${ }^{3,4}$

${ }_{25}$ Among the large variety of metal-organic frameworks, a remarkable number of compounds is based on the trimeric building unit $\left\{\mathrm{M}(\mathrm{III})_{3} \mathrm{O}(\mathrm{OH})\left(\mathrm{O}_{2} \mathrm{C}-\mathrm{R}\right)_{6}\left(\mathrm{H}_{2} \mathrm{O}\right)_{2}\right\}$ shown in Fig. $1 .^{5}$ This cluster has been known for a very long time, but the chemistry of MOFs shed a new light on its properties. Especially 30 the compounds known as MIL- $88^{3}$ (MIL stands for Materiaux de 1'Institute Lavoisier), MIL- $100^{6}$ and MIL-101 ${ }^{7}$, which are all based on this building unit, must be mentioned in this context. The materials known as M(III)-MIL-100 or M(III)-MIL-101 (M(III) designates the trivalent metal ion that forms the cluster) 35 exhibit permanent porosity with specific surface areas above $S_{\text {BET }}$ $=2000 \mathrm{~m}^{2} / \mathrm{g}$ and pore volumes up to $2 \mathrm{~cm}^{3} / \mathrm{g}$. Furthermore, the MOFs of the MIL-100-series [M(III) $\left.\left(\mu_{3}-\mathrm{O}\right)(\mathrm{OH})\left(\mathrm{H}_{2} \mathrm{O}\right)_{2}(\mathrm{BTC})_{2}\right]$ $(\mathrm{BTC}=1,3,5$-benzenetricarboxylate $)$ possess an outstanding thermal as well as chemical stability. MIL-100 could be obtained 40 up to now with the metal ions $\mathrm{V}^{3+8}, \mathrm{Cr}^{3+6}, \mathrm{Fe}^{3+}, \mathrm{Al}^{3+},{ }^{10}$ and $\mathrm{Sc}^{3+11}$, therefore giving an excellent example for isoreticular metal-organic frameworks. ${ }^{12}$ Besides the mentioned, desirable properties, the main interest in these materials originates from the coordination chemistry of the trimeric building unit. Since the

${ }_{45}$ charge of the cations is compensated by the $\mu_{3}$-oxide-ion, six carboxylate groups and one hydroxide ion, the weakly bond water molecules that complete the octahedral coordination geometry can be easily removed. ${ }^{13}$ In this way, coordinatively unsaturated sites (cus) can be created inside the framework. These are 50 available for coordination by other ligands ${ }^{14,15,16,17}$ or catalytic transformations. ${ }^{9,18,19}$

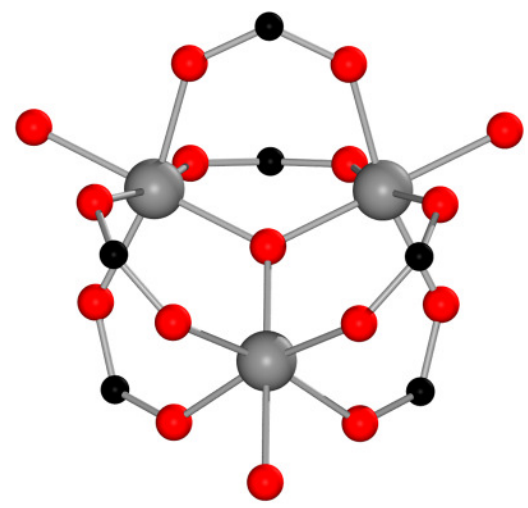

Fig. 1: The trimeric building unit observed in several MOFs based on $\mathrm{Sc}^{3+}, \mathrm{V}^{3+}, \mathrm{Cr}^{3+}, \mathrm{Fe}^{3+}$ or $\mathrm{Al}^{3+}$. M(III) atoms in grey, oxygen atoms in red, 55 carbon atoms in black.

Fe-ML-100 for example has been proven to be a versatile Lewisacidic catalyst. ${ }^{9}$ Furthermore, the trimeric cluster based on $\mathrm{Fe}^{3+}$ can be partially reduced to a mixed valency, which increases the number of cus. ${ }^{20}$ After this post-synthetic modification, the MOF 60 was successfully used for the separation of propane and propene. Furthermore it was successfully employed in the selective removal of N-heterocyclic aromatics from fuel feeds. ${ }^{21}$ Moreover, Fe-MIL-100 can be synthesised at very large scale, ${ }^{22}$ which makes it also a candidate for potential applications as water 65 adsorbent. ${ }^{23,24}$

Although this trimeric unit of the MIL-100 topology is known for manganese as a mixed valent cluster of two $\mathrm{Mn}^{3+}$ - and one $\mathrm{Mn}^{2+}$ ions $^{25}$, it has not been observed in the chemistry of MOFs yet. To the best of our knowledge, no MOF has been reported up to now 
based on inorganic nodes built up from $\mathrm{Mn}^{3+}$ and carboxylate ions, although $\mathrm{Mn}^{3+}$ was incorporated as part of the metal-organic linker molecule. ${ }^{26,27}$ Herein, we report the synthesis of the Mnbased analogue of the MIL-100 structure that could be obtained 5 using $\mathrm{Mn}(\mathrm{II})$ nitrate as the metal source. The divalent ions are oxidized in-situ in methanolic solution. The Mn-MIL-100 was fully characterised by TG-measurements, X-ray powder diffraction (XRPD), IR-spectroscopy and sorption experiments. To elucidate the crystallisation mechanism that comprises the 10 oxidation step, time dependent EDXRPD-data (energy dispersive $\mathrm{X}$-ray powder diffraction) under solvothermal conditions was recorded at the DESY-Synchrotron source in Hamburg. The evaluation of the data was conducted using two different kinetic models.

\section{${ }_{15}$ Experimental}

\section{Materials}

Methanol and ethanol (BASF, purum), trimesic acid $\left(\mathrm{H}_{3} \mathrm{BTC}\right.$, 1,3,5-benzenetricarboxylic acid) (Sigma) and $\mathrm{Mn}\left(\mathrm{NO}_{3}\right)_{2} \cdot 4 \mathrm{H}_{2} \mathrm{O}$ are commercially available and were used as obtained.

\section{${ }_{20}$ Synthesis}

Mn-MIL-100 (1) can be synthesised from a mixture of $400 \mathrm{mg}$ trimesic acid $(1.9 \mathrm{mmol})$ in $18 \mathrm{ml}$ methanol and $2 \mathrm{ml}$ of a $1 \mathrm{M}$ $\mathrm{Mn}\left(\mathrm{NO}_{3}\right)_{2} \cdot 4 \mathrm{H}_{2} \mathrm{O}$ solution in methanol $(2 \mathrm{mmol})$. The clear reaction solution was heated for $45 \mathrm{~min}$ at $125^{\circ} \mathrm{C}$ under stirring 25 in a closed glass reactor $(\mathrm{V}=100 \mathrm{~mL})$. After filtration, the obtained brown powder is redispersed in ethanol and filtered again. This final product is slightly sensitive to air exposure, but the crystallinity is retained for several hours and therefore it can be handled under non-inert conditions. It is noteworthy that the 30 quality of the XRPD patterns, especially the relative intensities up to $5^{\circ}$ (2theta), depends strongly on the amount of solvent molecules present in the pores.

\section{Characterisation}

The XRPD data was recorded on a Panalytical Xpert 35 diffractometer in reflection geometry (Cu- $\mathrm{K}_{\alpha 1}$-radiation). Temperature dependent XRPD data was collected on STOE Stadi $P$ diffractometer equipped with an IPPSD detector under air in capillaries. MIR spectra were recorded on an ATI Matheson Genesis spectrometer in the spectral range of 4000-400 $\mathrm{cm}^{-1}$ 40 using the $\mathrm{KBr}$ disk method. Thermogravimetric experiments were carried out under air on a NETZSCH-STA-409 CD thermal analyzer with a heating rate of $4 \mathrm{~K} / \mathrm{min}$. The sample was heated in an $\mathrm{Al}_{2} \mathrm{O}_{3}$ crucible under a flow of air $(25 \mathrm{ml} / \mathrm{min})$ and the data was corrected for buoyancy and current effects. Sorption 45 experiments were performed with a BELSORP Max from Bel Japan. INC.

\section{Results and Discussion}

\section{Structure}

${ }_{50}$ The structure of MIL-100 is based on the connection of the trimeric building units via trimesate anions (BTC). The inorganic bricks represent the corners of a so-called supertetrahedron (ST) and are connected by the tricarboxylate ions, which represent the faces of the tetrahedron.

55 The resulting framework corresponds to the zeotypic MTNnetwork, in which the supertetrahedra replace the tetrahedral nodes. This framework exhibits two kinds of very large pores with a diameter of $25 \AA$ and $29 \AA$ respectively. ${ }^{6}$ While the smaller cavity exhibits only pentagonal windows, the larger one 60 also possesses hexagonal apertures.

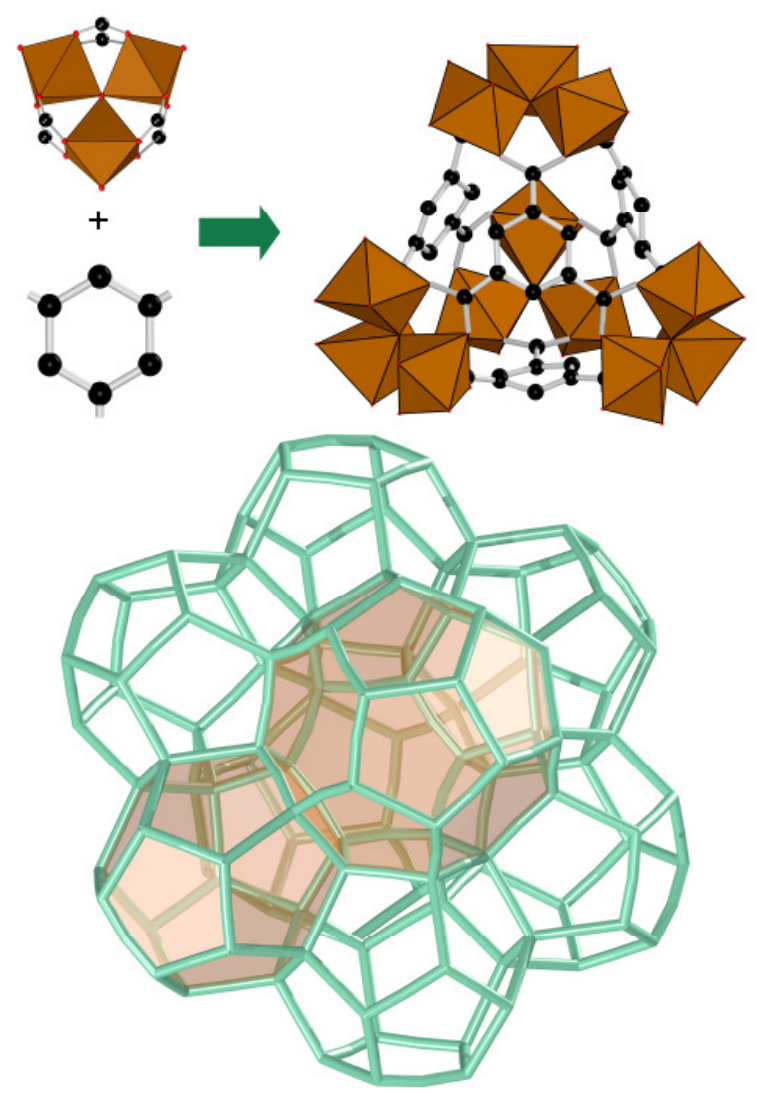

Fig. 2: Arrangement of the building units to form a supertetrahedron (top) and the MTN-framework with its two kinds of cavities emphasized in 65 brown (bottom). Crystal data taken from reference 6 .

The structure of $\mathbf{1}$ is very similar to the framework of the $\mathrm{Cr}$ MIL-100, as proven by XRPD (Fig. 3).

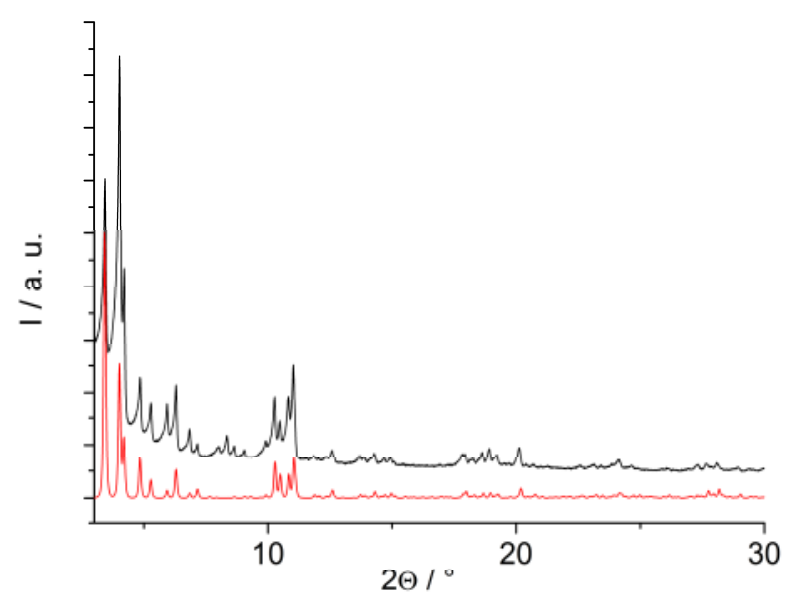

Fig. 3: Powder pattern for 1 (black) and simulated pattern for Cr-MIL101 (red). 
The lattice parameters are very similar (73.299(3) A and 72.906 $\AA$ for $\mathrm{Mn}-$ and Cr-MIL-100 respectively, see supporting informations Fig, S17) and the differences in relative intensities are mainly due to the occluded solvent molecules. Depending on 5 the amount and kind of solvent occluded inside the pores, the relative intensity of the reflections may strongly vary. The framework structure is stable in methanol as well as in ethanol. Upon solvent exchange with water, the crystallinity decreases substantially. The complete removal of the methanol and ethanol 10 from the pores by heating or by heating under vacuum $(0.1 \mathrm{mbar})$ results in an amorphization, while solvent removal under vacuum at room temperature retains the crystallinity (Fig. S1).

\section{Properties}

The TG-measurements in air indicate, that Mn-MIL-100 decomposes above $400^{\circ} \mathrm{C}$ in air (Fig. 4).

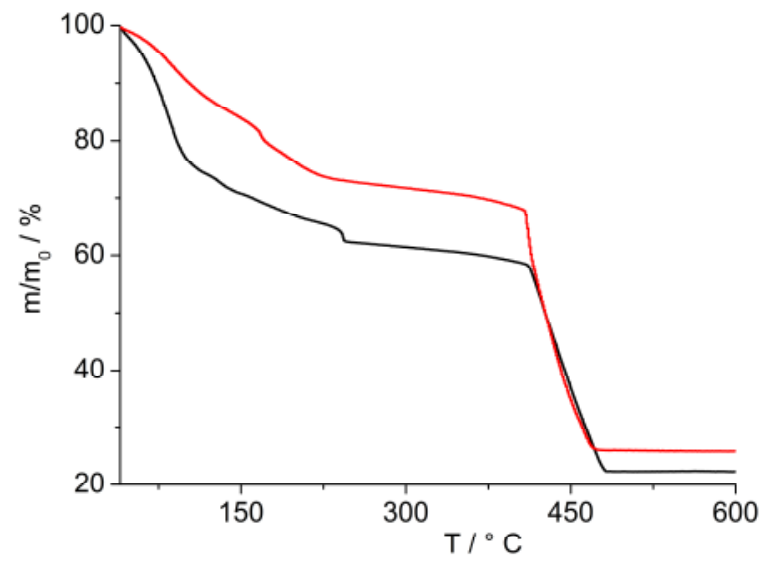

Fig. 4: TG-curves for the as synthesised material (black) and the Mn20 MIL-100 washed in ethanol (red).

The as synthesised (as) material (Fig. 4, black curve) shows a rapid loss of solvent starting with methanol removal at $40{ }^{\circ} \mathrm{C}$ and $\mathrm{H}_{2} \mathrm{O}$ above $75{ }^{\circ} \mathrm{C}$. The well defined step at $240{ }^{\circ} \mathrm{C}$, which we attribute to some residual acid (see also IR-spectrum, Fig. S3), ${ }_{25}$ can be easily removed by washing in ethanol. The curve for the washed material $\mathbf{1}$ in red shows a similar stepwise removal of ethanol followed by dehydration. Moreover, assuming the composition of the desolvated form of Mn-MIL-100 as $\left[\mathrm{Mn}_{3}(\mathrm{O})(\mathrm{BTC})_{2}\right]$, the decomposition above $400{ }^{\circ} \mathrm{C}$ is in very 30 good agreement with the theoretical value (ratio wht \% residue/ wht $\%$ at $400{ }^{\circ} \mathrm{C}=0.376$ (observed) and 0.384 (calculated)). The residual solid after complete decomposition was identified as the crystalline mixed valence manganese oxide $\mathrm{Mn}_{3} \mathrm{O}_{4}$ by XRPD measurements. Both results indicate that the trimeric building

35 unit in $\mathbf{1}$ exhibits most probably the mixed valency known from the corresponding trinuclear manganese carboxylate $\left[\mathrm{Mn}_{3} \mathrm{O}(\mathrm{MeOH})_{3}\left(\mathrm{O}_{2} \mathrm{C}-\mathrm{C}_{14} \mathrm{H}_{9}\right)_{6}\right] .{ }^{28}$ However, we were not able to unambiguously identify the oxidation state of the Mn-ions (Fig. S18, S19). Temperature dependent XRPD-measurements confirm 40 the amorphization upon solvent removal at elevated temperatures. The decrease in the crystallinity starts already at $120{ }^{\circ} \mathrm{C}$, indicated by peak broadening and lowering of the intensities. The amorphization is completed at $200{ }^{\circ} \mathrm{C}$.
The thermally activated amorphous as well as the vacuum treated 45 crystalline material were characterised regarding their sorption behaviour. In both cases a remarkable porosity was observed (Fig. S2 and Fig. 6, respectively). The capacity of the thermally activated materials $\left(0.1 \mathrm{mbar}, 4.5 \mathrm{~h}, 140{ }^{\circ} \mathrm{C}\right)$ strongly depends on the solvent treatment procedure. The micropore volumes were ${ }_{50}$ determined from the amount adsorbed at $\mathrm{p} / \mathrm{p}_{0}=0.5$. Starting from the as-material $\left(\mathrm{S}_{\mathrm{BET}}=1330 \mathrm{~m}^{2} / \mathrm{g}, \mathrm{V}_{\mathrm{Mic}}=0.59 \mathrm{~cm}^{3} / \mathrm{g}\right)$, the porosity is increased by activation in ethanol $\left(\mathrm{S}_{\mathrm{BET}}=1410 \mathrm{~m}^{2} / \mathrm{g}\right.$, $\mathrm{V}_{\text {Mic }}=0.63 \mathrm{~cm}^{3} / \mathrm{g}$ ). If the ethanol-washed Mn-MIL-100 is redispersed again in methanol, the exchange with the more 55 volatile solvent further increases the porosity $\left(\mathrm{S}_{\mathrm{BET}}=1610 \mathrm{~m}^{2} / \mathrm{g}\right.$, $\mathrm{V}_{\text {Mic }}=0.73 \mathrm{~cm}^{3} / \mathrm{g}$ ).

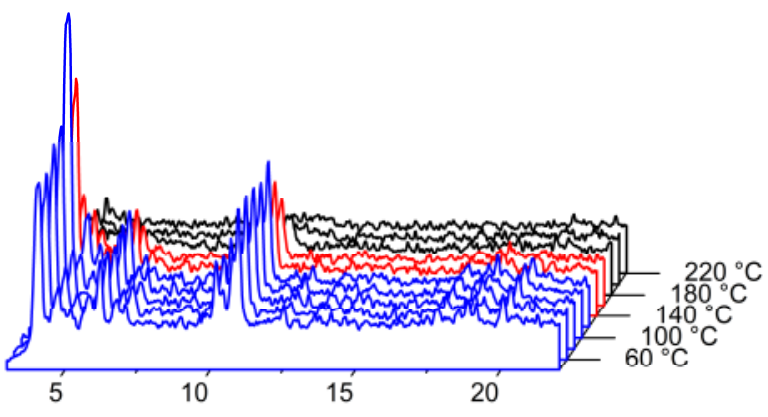

$2 \Theta /^{\circ}$

Fig. 5: Temperature dependent XRPD data for as synthesised Mn-MIL100 measured in a capillary under air.

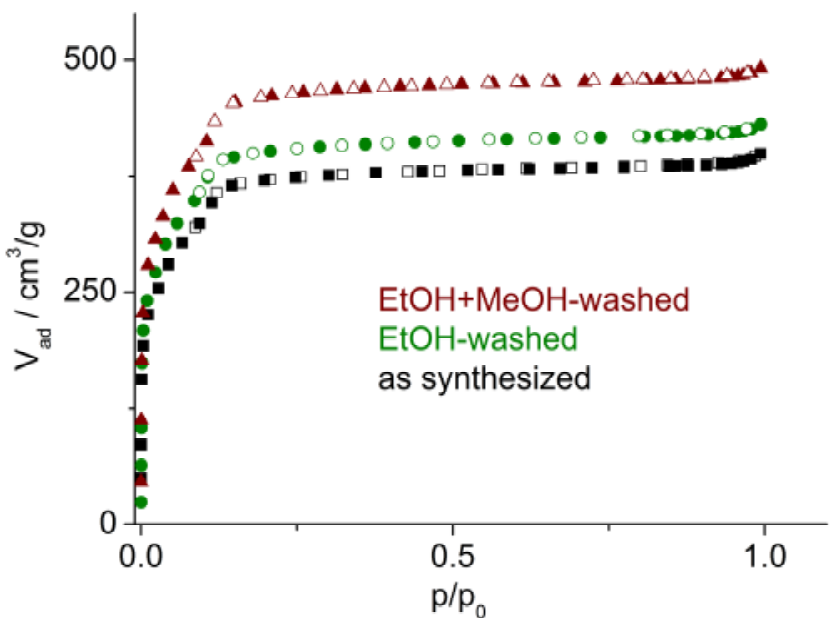

60 Fig. 6: $\mathrm{N}_{2}$ isotherms of thermally activated and solvent exchanged compounds measured at $77 \mathrm{~K}$. Filled symbols represent the adsorption, empty symbols represent the desorption branch.

Due to the amorphization of Mn-MIL-100 upon thermal solvent ${ }_{65}$ removal, the exact structure must be considered as unknown, but the steps in the sorption curves indicate, that the pore system is still topologically related to the MIL-100-framework. The slow increase in the sorption curve between $\mathrm{p} / \mathrm{p}_{0} \sim 0.01$ and 0.2 and the inflection point at $\mathrm{p} / \mathrm{p}_{0} \sim 0.15$ are characteristic for the filling of 70 large pores during the adsorption, and thus can be attributed to the adsorption into the larger cages of the framework. ${ }^{29}$ Interestingly, the sorption behaviour of the MOF activated only in vacuum is almost identical to the thermally activated MOF. 
Activation at room temperature at $\mathrm{p}=0.1 \mathrm{mbar}$ results in the preservation of the crystallinity (Fig. S1). This is probably due to the weakly bound solvent molecules at the trimeric unit that are not removed under vacuum at room temperature, while the 5 thermal treatment generates reactive cus, which destabilise the framework. The specific surface areas of the ethanol washed, vacuum-treated MOF is $1540 \mathrm{~m}^{2} / \mathrm{g}$ and its micropore volume is $0.67 \mathrm{~cm}^{3} / \mathrm{g}$. However, the MOF activated in vacuum at room temperature as well as the compound that was thermally activated 10 decompose within few hours after re-exposure to air and lose their porosity.

\section{Reaction Mechanism}

15 To the best of our knowledge, the in-situ formation of $\mathrm{Mn}^{3+}$ ions by oxidation of $\mathrm{Mn}^{2+}$ ions in the synthesis of MOFs has not been described previously. Therefore, the reaction conditions were investigated in detail. The crucial role can be attributed to the use of $\mathrm{Mn}\left(\mathrm{NO}_{3}\right)_{2} \cdot 4 \mathrm{H}_{2} \mathrm{O}$. Employing the chloride or the acetate of ${ }_{20} \mathrm{Mn}$ (II) under otherwise identical reaction conditions, we were not able to observe any oxidized Mn-species. The direct use of the trivalent acetate did also not lead to the formation of $\mathbf{1}$. Furthermore, the oxidation is independent of the atmosphere in the reactor. Purging the solution with argon followed by sealing 25 of the reactor under inert conditions did not affect the product formation. The use of other organic acids like nitroterephthalic acid or pyridine-dicarboxylic acid also led to the oxidation of Mn(II), but no crystalline products could be obtained yet. A similar result was observed when the solvent was changed from

30 methanol to another solvent. Above certain temperatures, the oxidation of the $\mathrm{Mn}(\mathrm{II})$ was also observed in ethanol or water employing manganese nitrate, but no Mn-MIL-100 was obtained. In contrast to this, we did not observe any oxidation in $\mathrm{N}, \mathrm{N}$-dimethylformamide as the solvent. Based on standard 35 potentials, the oxidation of methanol by $\mathrm{Mn}^{3+}$ ions is expected, but the described solvothermal system seems to be unique in its ability to "capture" and stabilize the Mn(III) in its trivalent state, prior to the formation of $\mathbf{1}$.

The rather short reaction time $(45 \mathrm{~min})$ that leads to the formation 40 of Mn-MIL-100 makes it a perfectly suitable system for a kinetic evaluation of the crystallization process using synchrotron radiation. ${ }^{30}$ While the synthesis of hybrid compounds often takes days, in this case the crystallisation already starts after ten minutes, as observed by clouding of the reactant solution. Thus, 45 in-situ energy-dispersive XRPD measurements were carried out at the beamline F3 of the HASYLAB at DESY (Hamburg, Germany). The set-up that was used for the EDXRPD measurements allowed us to incorporate a microwave-oven into the beam as recently described by our group. ${ }^{31}$ Polychromatic 50 white synchrotron light is used and the energy of the diffracted $\mathrm{X}$-rays is recorded under a constant detector angle by a Gedetector. The reactions were carried out in small vials $\left(\mathrm{V}_{\mathrm{Max}}=5\right.$ $\mathrm{mL}$, filling degree $3 \mathrm{~mL}$ ) using a Biotage Initiator MW-oven. Time-dependent EDXRPD patterns were recorded at three ${ }_{55}$ different temperatures $\left(125^{\circ} \mathrm{C}, 135^{\circ} \mathrm{C}\right.$ and $\left.145^{\circ} \mathrm{C}\right)$ with a time resolution of 30 seconds. A typical set of spectra is shown in Figure 7.

The integration of the peak area corresponds to the reaction progress $\alpha$, and was used in its normalized form to study the 60 kinetics of crystallisation. In this study the peak corresponding to the 113 reflection, which is the one with the highest intensity, was chosen. The plot of the reaction progress for the different temperatures is shown in Fig. 8.

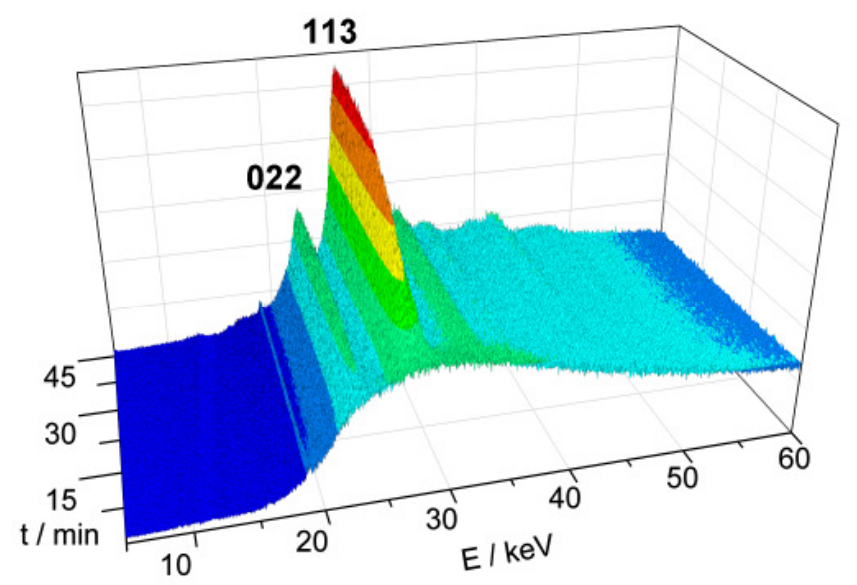

65 Fig. 7: EDXRPD-spectra for the reaction at $135^{\circ} \mathrm{C}$. The strongest peaks are given with their hkl-indices.

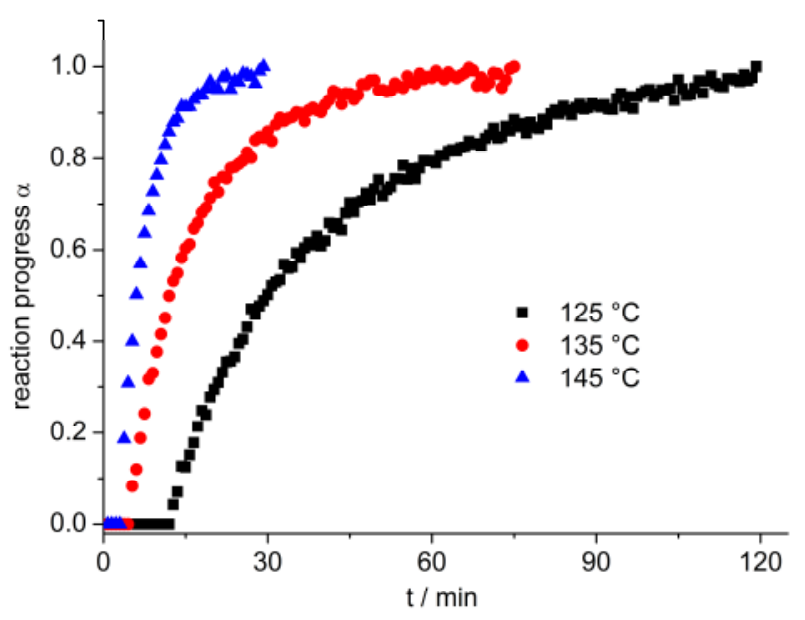

Fig. 8: Reaction progress for the synthesis of Mn-MIL-100 at the three different temperatures depending on the synthesis time.

Useful information can be directly extracted from these plots. The induction time $\left(t_{\text {ind }}\right)$, which is the period during which no crystalline products are observed, is shortened for the synthesis at higher temperatures (at $125{ }^{\circ} \mathrm{C}$ : 12 minutes,; at $135{ }^{\circ} \mathrm{C}$ : 4.5 75 minutes; at $145{ }^{\circ} \mathrm{C}$ : 3 minutes). Furthermore, the reaction is completed after shorter times at higher temperatures. While the crystallisation is finished after 30 minutes at $145^{\circ} \mathrm{C}$, the reaction is slowed down at $135{ }^{\circ} \mathrm{C}$ ( 75 minutes) and $125^{\circ} \mathrm{C}(120$ minutes $)$. Further information can be gained by careful evaluation of the 80 data. Two models are commonly employed to evaluate the crystallisation. The most frequently used method is the SharpHancock analysis. ${ }^{32}$ According to this model, the plot of $\ln [-\ln (1-$ $\alpha)$ ] over $\ln \left(t_{\text {Red }}\right)$ yields a straight line with the slope $m$ and the intercept $m \ln (k)$. Here, $\mathrm{t}_{\text {Red }}=\mathrm{t}-\mathrm{t}_{\text {ind }}$ and $m$ is the Avrami exponent, 85 which gives information about the reaction mechanism. In the 
case of Mn-MIL-100, plotting $\ln [-\ln (1-\alpha)]$ vs. $\ln \left(t_{\text {Red }}\right)$ leads to two linear sections with different slopes (Fig. S4-S9). Thus, two dominating mechanisms are taking place during the crystallisation of Mn-MIL-100, which was already reported in 5 other studies. ${ }^{33}$ Therefore, the Sharp-Hancock analysis was carried out for both linear sections. The final values are summarized in Tab. 1.

Table 1 Rate constants $\left(\mathrm{k}_{1}\right.$ and $\left.\mathrm{k}_{2}\right)$, Avrami exponents $\left(\mathrm{m}_{1}\right.$ and $\left.\mathrm{m}_{2}\right)$ and corresponding reaction periods, evaluated according to the Sharp10 Hancock model.

\begin{tabular}{c|c|c|c|c|c|c}
\hline $\mathrm{T}\left[{ }^{\circ} \mathrm{C}\right]$ & $k_{1}\left[\mathrm{~min}^{-1}\right]$ & $m_{1}$ & $\mathrm{t}_{\text {Red }}[\mathrm{min}]$ & $k_{2}\left[\mathrm{~min}^{-1}\right]$ & $m_{2}$ & $\mathrm{t}_{\text {Red }}[\mathrm{min}]$ \\
\hline 125 & $0.048(10)$ & $1.97(17)$ & $0-5$ & $0.030(1)$ & $1.009(8)$ & $5-120$ \\
\hline 135 & $0.109(6)$ & $1.77(8)$ & $0-3$ & $0.074(4)$ & $0.91(2)$ & $3-75$ \\
\hline 145 & $0.177(11)$ & $1.66(8)$ & $0-3$ & $0.189(20)$ & $0.90(4)$ & $3-30$
\end{tabular}

Both rate constants increase with temperature. The Avrami exponents for the first stage of the reaction indicate $\left(1.66(8) \leq \mathrm{m}_{1}\right.$ $\leq 1.97(17)$ ) that during the first minutes, the crystallisation 15 mechanism is controlled by nucleation (theory, $\mathrm{m}=2$ ). During the second period of the reaction Avrami exponents are in the range of 0.90(4) to 1.009(8), which are characteristic for a first order reaction mechanism. This could be due to the in-situ generation of $\mathrm{Mn}^{3+}$ ions, which should then be the rate limiting 20 step. Thus, the Sharp-Hancock model shows that the reaction can be separated in two parts that are dominated by different crystallisation mechanisms.

The second, recently more often used model to evaluate the extent of crystallisation is the Gualtieri method. ${ }^{34}$ This model

25 takes into account, that the nucleation and the crystal growth are two separate processes, represented in equation 1.

$$
\alpha=\frac{1}{1+\exp \left[-\left(\frac{t-a}{b}\right)\right]}\left\{1-\exp \left[-\left(k_{g} t\right)^{n}\right]\right\}
$$

The reaction extent or progress $\alpha$ is the same as in the SharpHancock model, $t$ is the time, $k_{g}$ is the rate constant for the crystal 30 growth, $a=1 / k_{n}$, the reciprocal rate constant of the nucleation, $b$ is a constant also related to nucleation and $n$ is the dimensionality of the crystal growth.

Since previous measurements for isoreticular compounds have shown that the crystals of MIL-100 are mostly octahedral, ${ }^{10}$ the

35 dimensionality of the crystal growth was set to $n=3$. The results of the fits are shown in Fig. 9, S10, S11 and the parameters are summarized in Table 2.

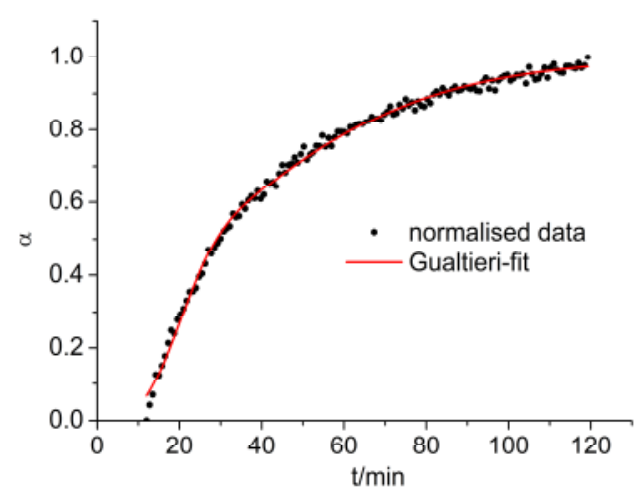

Fig. 9 Gualtieri-fit for the normalised data measured at $125^{\circ} \mathrm{C}$.

40 Table 2 Rate constants and nucleation parameter $b$ as obtained by using the Gualtieri model.

\begin{tabular}{c|c|c|c}
\hline $\mathrm{T}\left[{ }^{\circ} \mathrm{C}\right]$ & $k_{\mathrm{n}}\left[\mathrm{min}^{-1}\right]$ & $k_{\mathrm{g}}\left[\mathrm{min}^{-1}\right]$ & $b\left[\mathrm{~min}^{-1}\right]$ \\
\hline 125 & $0.039(1)$ & $0.0486(4)$ & $26.1(4)$ \\
\hline 135 & $0.116(6)$ & $0.112(1)$ & $12.6(3)$ \\
\hline 145 & $0.241(25)$ & $0.203(7)$ & $4.7(3)$
\end{tabular}

The rate constants for nucleation and growth increase with temperature. Moreover, the values are in the same order of 45 magnitude as the ones obtained from the Sharp-Hancock model.

In comparison with the results of crystallisation studies on other hybrid compounds ${ }^{35,36,37,38}$ as well as inorganic materials ${ }^{39,40,41,42}$ the rate constants and coefficients are not drastically different. Thus the in situ oxidation of $\mathrm{Mn}^{2+}$ does not result in uncommon 50 crystallisation kinetics.

The constants that are determined by the Gualtieri-method can be used to calculate the probability of nucleation $\mathrm{P}_{\mathrm{N}}$ which is

$$
P_{N}=e^{-\frac{(t-a)^{2}}{b^{2}}}
$$

The plot of probability of nucleation versus time shows, that an 55 increasing temperature leads to a faster nucleation with an earlier maximum, while the later times of the reaction are dominated by crystal growth and the probability of nucleation becomes almost negligible (Fig. 10).

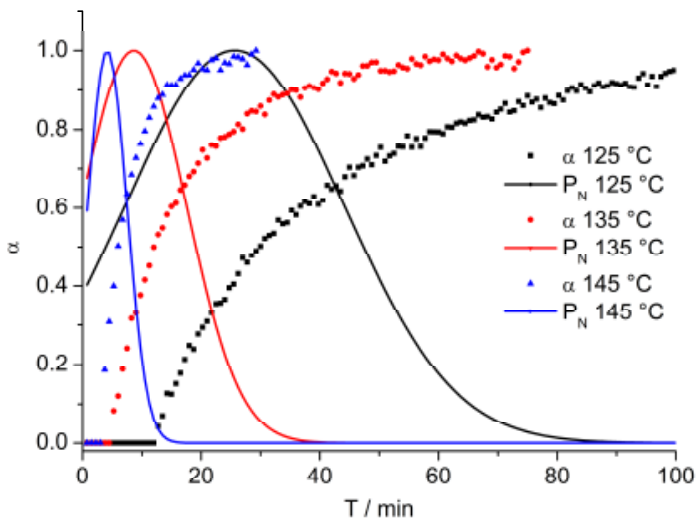

60 Fig. 10 The reaction progress $\alpha$ (symbols) and the probability of nucleation $\mathrm{P}_{\mathrm{N}}$ (solid line) as determined for the different temperatures $\left(125^{\circ} \mathrm{C}\right.$ : black; $135^{\circ} \mathrm{C}$ : red; $145^{\circ} \mathrm{C}$ : blue). 
The Arrhenius activation energy for the reaction can be obtained for both reaction steps by plotting $\ln (k)$ vs $1 / \mathrm{T}$ (Fig. S12-S15). These values are summarised in Tab. 3 .

Table 3 Activation energies evaluated from the different reaction stages 5 for the Sharp-Hancock and the Gualtieri model.

\begin{tabular}{ccccc} 
model & \multicolumn{2}{c}{ Sharp-Hancock } & \multicolumn{2}{c}{ Gualtieri } \\
rate constant & $\mathrm{k}_{1}$ & $\mathrm{k}_{2}$ & $\mathrm{k}_{\mathrm{n}}$ & $\mathrm{k}_{\mathrm{g}}$ \\
$\mathrm{E}_{\mathrm{a}}[\mathrm{kJ}]$ & $89.8 \pm 11.7$ & $127.0 \pm 3.0$ & $126.5 \pm 12.4$ & $98.9 \pm 8.4$
\end{tabular}

The activation energies are all in the same order of magnitude and comparable to the values reported previously for MOFs (CAU-1: 131-136 kJ, ${ }^{36}$ MOF-14: 64-83 kJ, ${ }^{35}$ $\left.{ }_{10} \mathrm{Li}_{4}\left[\mathrm{C}_{4} \mathrm{H}_{2} \mathrm{~S}\left(\mathrm{CO}_{2}\right)_{2}\right]_{2}\left[\mathrm{C}_{3} \mathrm{H}_{7} \mathrm{NO}\right]_{2}: 114-132 \mathrm{~kJ},{ }^{43}\right)$.

\section{Conclusions}

The accessibility of a Mn(III)-based MOF exhibiting permanent porosity has been demonstrated in this study. Since the structure of Mn-MIL-100 is composed of trimeric units of corner-sharing 15 octahedra $\left\{\mathrm{Mn}_{3}\left(\mu_{3}-\mathrm{O}\right)\left(\mathrm{H}_{2} \mathrm{O}\right)_{3}\left(\mathrm{O}_{2} \mathrm{C}-\mathrm{R}\right)_{6}\right\}$ the formation of other MOFs which are also based on this inorganic building units could be possible.

\section{Acknowledgements}

The help of Dr. Andre Rothkirch (HASYLAB, DESY Hamburg) 20 and Mark Feyand (CAU Kiel) during the recording and interpretation of the EDXRPD-data is gratefully acknowledged. The research leading to these results has received funding from the European Community's Seventh Framework Programme (FP7/2007-20013) under grant agreement n 228862".

\section{${ }_{25}$ Notes and references}

${ }^{a}$ Institute of Inorganic Chemistry, Christian-Albrechts-Universität Kiel, Max-Eyth-Straße 2, 24118 Kiel, Germany; stock@ac.uni-kiel.de $\dagger$ Electronic Supplementary Information (ESI) available: IR-spectrum, XRPD-data and sorption isotherm for the vacuum-treated MOF, Sharp30 Hancock-plots and Fits, Gualtieri-Fits, Arrhenius.plots. See DOI: 10.1039/b000000x/

N. Stock, S. Biswas, Chem. Rev., 2012, 112, 933.

H. K. Chae, D. Y. Siberio-Perez, J. Kim, Y. Go, M. Eddaoudi, A. J. Matzger, M. O'Keeffe, O. M. Yaghi, Science, 2004, 427, 523.

C. Serre, C. Mellot-Draznieks, S. Surble, N. Audebrand, Y. Filinchuk, G. Ferey, Science, 2007, 315, 1828.

4 M. I. H. Mohideen, B. Xiao, P. S. Wheatley, A. C. McKinlay, Y. Li, A. M. Z. Slawin, D. W. Aldous, N. F. Cessford, T. Düren, X. Zhao, R. Gill, K. M. Thomas, J. M. Griffin, S. E. Ashbrook, R. E. Morris, Nature Chemistry, 2011, 3, 304.

C. T. Dziobkowski, T. J. Wrobleski, D. B. Brown, Inorg. Chem., 1981, 20, 671 .

6 G. Ferey, C. Serre, C. Mellot-Draznieks, F. Millange, S. Surble, J. Dutour, I. Margiolaki, Angew. Chem. Int. Edt. 2004, 43, 6296. G. Férey, C. Mellot-Draznieks, C. Serre, F. Millange, J. Dutour, S. Surblé, I. Margiolaki, Science, 2005, 23, 2040.; E. Stavitski, M. Goesten, J. Juan-Alcaniz, A. Martinez-Joaristi, P. Serra-Crespo, A. V. Petukhov, J. Gascon, F. Kapteijm, Angew. Chem. Int. Ed., 2011, 50, 9624.

8 A. Lieb, H. Leclerc, T. Devic, C. Serre, I. Margiolaki, F. Mahjoubi, J. S. Lee, A. Vimont, M. Daturi, J.-S. Chang, Micropor. Mesopor. Mater., 2012, 157, 18.

9 P. Horcajada, S. Surble, C. Serre, D.-Y. Hong, Y.-K. Seo, J.-S. Chang, J.-M. Greneche, I. Margiolaki, G. Ferey, Chem. Commun., 2007, 27, 2820.
10 C. Volkringer, D. Popov, T. Loiseau, G. Férey, M. Burghammer, C. Riekel, M. Haouas, F. Taulelle, Chem. Mater., 2009, 21, 5695.

11 J. P. S. Mowat, S. R. Miller, A. M. Z. Slawin, V. R. Seymour, S. E. Ashbrook, P. A. Wright, Micropor. Mesopor. Mater., 2011, 142, 322.

12 S. H. Jhung, N. A. Khan, Z. Hasan, CrystEngComm, 2012, 14, 7099.

13 M. Haouas, C. Volkringer, T. Loiseau, G. Ferey, F. Taulelle, J. Phys. Chem. C, 115, 17934.

14 D.-Y. Hong, Y. K. Hwang, C. Serre, G. Ferey, J.-S. Chang, Adv. Funct. Mater., 2009, 19, 1537.

15 H. Leclerc, A. Vimont, J.-C. Lavalley, M. Daturi, A. D. Wiersum, P. L. Llwellyn, P. Horcajada, G. Ferey, C. Serre, Phys. Chem. Chem. Phys., 2011, 13, 11748.

16 L. Hamon, C. Serre, T. Devic, T. Loiseau, F. Millange, G. Ferey, G. De Weireld, J. Am. Chem. Soc., 2009, 131, 8775.

17 C. Volkringer, H. Leclerc, J.-C. Lavalley, T. Loiseau, G. Férey, M. Daturi, A. Vimont, J. Phys. Chem. C, 2012, 116, 5710.

18 L. Kurfirtova, Y.-K. Seo, Y. K. Hwang, J.-S. Chang, J. Cejka, Catalysis Today, 2012, 179, 85.

19 F. Vermoortele, R. Ameloot, L. Alaerts, R. Matthessen, B. Carlier, E. V. Ramos Fernandez, J. Gascon, F. Kapteijn, D. E. De Vos, J. Mater. Chem., 2012, 22, 10313.

20 J. W. Yoon, Y.-K. Seo, Y. K. Hwang, J.-S. Chang, H. Leclerc, S. Wuttke, P. Bazin, A. Vimont, M. Daturi, E. Bloch, P. L. Llewellyn, C. Serre, P. Horcajada, J.-M. Greneche, A. E. Rodrigues, G. Ferey, Angew. Chem. Int. Edt., 2010, 49, 5949.

21 M. Maes, M. Trekels, M. Boulhout, S. Schouteden, F. Vermoortele, L. Alaerts, D. Heurtaux, Y.-K. Seo, Y. K. Hwang, J.-S. Chang, I. Beurroies, R. Denoyel, K. Temst, A. Vantomme, P. Horcajada, C. Serre, D. E. De Vos, Angew. Chem. Int. Edt., 2011, 50, 4210.

22 Y.-K. Seo, J. Woong Yoon, J. Sun Lee, U-H. Lee, Y. K. Hwang, C.H. Jun, P. Horcajada, C. Serre, J.-S. Chang, Micropor. Mesopor. Mater., 2012, 157, 137.

23 Y.-K. Seo, J. W. Yoon, J. S. Lee, Y. K. Hwang, C.-H. Jun, J.-S. Chang, S. Wuttke, P. Bazin, A. Vimont, M. Daturi, S. Bourrelly, P. L. Llewellyn, P. Horcajada, C. Serre, G. Férey, Adv. Mater., 2012, 24, 806 .

24 F. Jeremias, A. Khutia, S. K. Henninger, C. Janiak, J. Mater. Chem., 2012, 22, 10148.

25 T. Lis, V- Kinzhybalo, K. Zieba, Acta Cryst., 2005, E61, 2382.

26 F. Song, C. Wang, J. M. Falkowski, L. Ma, W. Lin, J. Am. Chem. Soc., 2010, 132, 15390.

27 X.-L. Yang, M.-H. Xie, C. Zou, Y. He, B. Chen, M. O'Keeffe, C.-D. Wu, J. Am. Chem. Soc., 2012, 134, 10638.

28 N. Taleb, V. J. Richards, S. P. Argent, J. van Slageren, W. Lewis, A. J. Blake, N. R. Champness, Dalton Trans., 2011, 40, 5891.

29 A. Sonnauer, F. Hoffmann, M. Fröba, L. Kienle, V. Duppel, M. Thommes, C. Serre, G. Ferey, N. Stock, Angew. Chem. Int. Edt., 2009, 48, 3791.

30 N. Pienack, W. Bensch, Angew. Chemie Int. Ed, . 2011, 50, 2014.

31 M. Feyand, C. Näther, A. Rothkirch, N. Stock, Inorg. Chem., 2010, 49, 11158.

32 J. D. Sharp, J. H. Hancock, J. Am. Ceram. Soc., 1972, 55, 74.

33 N. Pienack, C. Näther, W. Bensch, Eur. J. Inorg. Chem., 2009, 937

34 A. F. Gualtieri, Phys. Chem. Miner., 2001, 28, 719.

35 F. Millange, R. El Osta, M. E. Medina, R.I. Walton, CrystEng Comm. 2011, 13, 103.

36 T. Ahnfeldt, J. Moellmer, V. Guillerm, R. Staudt, C. Serre, N. Stock, Chem.-Eur. J., 2011, 17, 6462.

37 F. Millange, M. E. Medina, N. Guillou, G. Ferey, K. M. Golden, R. I. Walton, Angew. Chem. Int Ed., 2010, 49, 763.

38 E. Stavitski, M. Goesten, J. Juan-Alcaniz, A. Martinez-Joaristi, P. Serra-Crespo, A. V. Petukhov, J. Gascon, F. Kapteijn, Angew. Chem. Int. Ed., 2011, 50, 9624.

39 R. J. Francis, S. O'Brien, A. M. Fogg, P. Shiv Halasyamani, D. O'Hare, T. Loiseau, G. Ferey, J. Am. Chem. Soc., 1999, 121, 1002.

40 R. Kiebach, N. Pienack, M.-E. Ordolff, F. Studt, W.Bensch, Chem. Mater. 2006, 18, 1196.

41 Y. Zhou, E. Antonova, W. Bensch, G. R. Patzke, Nanoscale, 2010, 2, 2412.

42 Y. Zhou, E. Antonove, Y. Lin, J.-P. Grunwaldt, W. Bensch, G. R. Patzke, Eur. J. Inorg. Chem. 2012, 783. 
43 R. El Osta, M.Frigoli, J.Marrot, M.E. Medina, R.I. Walton, F. Millange, Cryst. Growth Des., 2012, 12, 1531. 